\title{
Echocardiographic analysis of left ventricular function in term and preterm neonates at week 40 of postconceptional life
}

\author{
Renata Bokiniec', Paweł Własienko², Joanna Szymkiewicz-Dangel³, Maria K. Borszewska-Kornacka \\ 1 Neonatal and Intensive Care Department, Medical University of Warsaw, Warsaw, Poland \\ 2 Perinatal Cardiology Unit, Medical University of Warsaw, Warsaw, Poland \\ 3 Department of Perinatal Cardiology and Congenital Anomalies, Centre of Postgraduate Medical Education, Warsaw, Poland
}

\section{KEY WORDS}

echocardiography, left ventricle, preterm neonate, pulsed-wave Doppler imaging, tissue Doppler imaging

\section{EDITORIAL}

page 417
Correspondence to: Renata Bokiniec, MD, PhD, Department of Neonatal and Intensive Care, Medical University of Warsaw, ul. Karowa 2, 00-315 Warszawa, Poland, phone: +48225966155, email: r.bokiniec@wp.pl Received: January 13, 2019. Revision accepted: February 27, 2019 Published online: February 27, 2019. Kardiol Pol. 2019; 77 (4): 445-450 doi:10.5603/KP.a2019.0040 Copyright by Polskie Towarzystwo Kardiologiczne, Warszawa 2019

\begin{abstract}
BACKGROUND Both gestational and chronological age of the neonate may influence and impair the function of the delicate and immature myocardium. However, the transition from fetal to neonatal circulation in preterm neonates is poorly understood.
\end{abstract}

AIMS This study aimed to compare left ventricular (LV) systolic and diastolic function between premature neonates at expected term and term neonates during the postnatal cardiovascular transitional period. METHODS Using echocardiography, we assessed systolic and diastolic function of the LV in 89 preterm neonates at week 40 of postconceptional age and 29 term neonates after closure of the patent ductus arteriosus (PDA) and on the 28th day of life. Based on M-mode images, we measured myocardial thickness and fractional shortening (FS\%). Using pulsed-wave Doppler echocardiography, we estimated cardiac output, myocardial performance index (MPI), and LV diastolic function (E and A waves, E/A ratio). Systolic and diastolic function was also assessed by tissue Doppler imaging.

RESULTS Compared with term neonates on the 28th day of life, preterm neonates had reduced myocardial thickness $(P \leq 0.04), \mathrm{FS} \%(P=0.002)$, and cardiac output $(P=0.01)$. However, preterm neonates had a lower MPI than term neonates after PDA closure $(P<0.001)$ and on the 28 th day of life $(P=0.02)$. The E/A ratio and $S^{\prime}$ wave values were similar in preterm and term neonates $(P>0.05)$.

CONCLUSIONS Preterm neonates at 40 weeks of postconceptional age have preserved systolic and diastolic function of the LV.

INTRODUCTION The transition from fetal to neonatal circulation is complex and involves many changes to the circulatory system during the first weeks of life. After birth, the umbilical vessels are clamped, and major fetal shunts, like the ductus arteriosus, ductus venosus, and foramen ovale, close. These changes enable adaptation to the ex utero environment, including sufficient blood flow through the lungs to support oxygenation. ${ }^{1}$ However, this transition is challeng-ing for the circulatory system. For example, after birth, the low-resistance circulation through the placenta changes to systemic circulation, with increased systemic blood pressure and left ventricular (LV) afterload, whereas the fetal pulmonary circulation changes from a high-resistance and low-flow to a low-resistance and high-flow circulation, with increased ventricular preload. ${ }^{2-5}$ Moreover, the closure of fetal shunts and an increased oxygen demand after birth nearly double cardiac output. ${ }^{6}$

The transition from fetal to neonatal circulation puts a high strain on the myocardium in children born at term and is even more stressful for the myocardium in preterm neonates. For example, in preterm neonates, the proliferation of cardiomyocytes is reduced compared with age-matched controls, which may make 


\section{WHAT'S NEW?}

The immature heart in preterm neonates completes its development ex utero, which may result in impaired myocardial function. The transition from fetal to neonatal circulation involves substantial changes, such as the closure of major shunts. Therefore, impaired myocardial function in preterm neonates could increase the risk of hemodynamic instability in the fetal-to-neonatal transitional period. In this study, we used several echocardiographic measurements to compare systolic and diastolic function of the left ventricle between preterm neonates and neonates born at full term. We found that preterm neonates had normal left ventricular systolic and diastolic function. Although preterm neonates had reduced fractional shortening and cardiac output compared with term neonates, these differences were likely due to a smaller heart size in preterm neonates. Therefore, we conclude that the myocardium in preterm neonates has preserved functional capacity.

the neonates more vulnerable to circulatory collapse. ${ }^{7}$ Preterm neonates often have persistent patent ductus arteriosus (PDA), which increases $\mathrm{LV}$ volume overload and leads to enlargement of the LV and left atrium and to mitral valve insufficiency. ${ }^{8}$ Moreover, left-to-right flow through persistent PDA, which increases blood flow through pulmonary circulation and causes ductal steal, is associated with systemic hypotension. ${ }^{9}$ On the other hand, sudden PDA closure with surgical ligation reduces volume overload and LV cardiac output, which often necessitates transient vasopressor support. ${ }^{10}$ Bronchopulmonary dysplasia, which is frequent in preterm neonates, also impairs LV function, particularly diastolic function (early and atrial mitral inflow). ${ }^{11}$

Although preterm neonates are at an increased risk of hemodynamic instability in the postnatal circulatory transition period, we know little about LV function and potential differences in heart function between preterm and term neonates during this critical period. This lack of knowledge is partly because it is difficult to decide at which time point such comparisons should be performed. For example, comparing heart function between preterm and term neonates matched for postconceptional age (PCA) may not be valid, because the circulatory system in preterm neonates has had more time to adapt to the external environment than in term neonates at the same PCA. Therefore, we decided to compare LV function between preterm neonates at expected term (ie, week 40 of PCA) and term neonates at 2 time points: after PDA closure and on the 28th day of life (ie, after substantial adaptations in the circulatory system had occurred).

METHODS Participants We included 89 preterm neonates and 29 neonates born at full term, who served as a control group. The study was approved by the Bioethics Committee of the Medical University of Warsaw. Parents or legal guardians of all neonates included in the study signed informed consent.
Echocardiography In the premature neonates, echocardiography was performed at week 40 of PCA. In the control group, it was performed after PDA closure (ie, between the 3rd and 6th day of life) and at the end of the neonatal period (ie, on the 28th day of life). For all measurements, we used the HD 15 XE ultrasound system (Philips N.V., Amsterdam, the Netherlands) equipped with a 12-MHz sector probe. All echocardiography examinations were performed at bedside by one investigator. Left ventricular function parameters were calculated offline with the Image Arena software (TomTec, Unterschleißheim, Germany).

M-mode echocardiography Based on M-mode images, we measured the interventricular septum thickness (IVSd), LV posterior wall thickness (LVPWd), and LV internal diameter at end diastole (LVIDd) in the parasternal long-axis view at the level of the mitral valve leaflets. To measure LV systolic function, we calculated the fractional shortening (FS\%) in the parasternal long-axis view as follows: FS\% = LV end--diastolic diameter - LV end-systolic diameter / LV end-diastolic diameter $\times 100 \%$.

Pulsed-wave Doppler To estimate LV systolic function, we measured the LV cardiac output in the apical 5-chamber view as follows: LV cardiac output $(\mathrm{ml} / \mathrm{min})=$ velocity time integral $\times$ aortic area $\times$ heart rate. The velocity time integral was defined as the aortic stroke distance, and the aortic area was calculated based on the aortic diameter in the parasternal long-axis view.

We assessed LV diastolic function with the peak early diastolic (E) wave, peak late diastolic (atrial A) wave, and the mitral E/A ratio. All measurements were done in the apical 4-chamber view.

To assess the overall LV function (ie, systolic and diastolic), we calculated the myocardial performance index (MPI). To measure LV MPI, a Doppler sample volume was placed below the mitral valve towards the ventricular septum with the pulsed-wave Doppler tracing including both the E/A (positive) and the aortic (negative) blood flow waveforms. The isovolumetric contraction time (ICT), isovolumetric relaxation time (IRT), and ejection time (ET) were measured. The MPI was calculated as follows: (isovolumetric contraction time + isovolumetric relaxation time) / ejection time (FIGURE1).

Tissue Doppler imaging We used tissue Doppler imaging (TDI) to evaluate systolic and diastolic function of the LV. We measured myocardial velocity in the apical 4-chamber view with colorcoded TDI. A spectral Doppler gate was placed in the center of the LV muscle below the lateral mitral annulus, so that the angle of the ultrasound beam was less than $30^{\circ}$. The cardiac cycle was analyzed as 3 flow waves: systolic S' wave, 


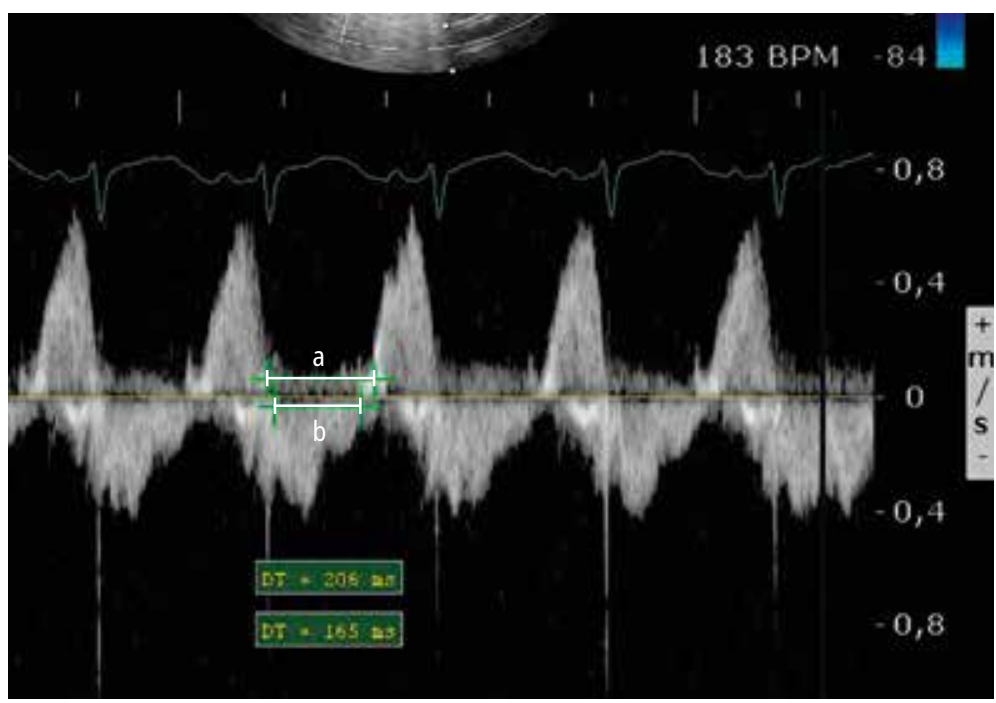

FIGURE 1 Pulsed Doppler tracing in a neonate: calculation of myocardial performance index (MPI). The MPI was calculated as follows: MPI $=a-b / b$ (see the Methods section for full description). of preterm with term neonates. A $P$ value of less than 0.05 was considered significant. All calculations were done in the Statistica software (StatSoft Inc., Tulsa, Oklahoma, United States).

RESULT Patient characteristics We included 89 preterm neonates and 29 neonates born at full term. The baseline clinical characteristics of all neonates are shown in TABLE 1 . The preterm neonates had a higher heart rate than the term neonates after PDA closure $(P<0.001)$, but not on the 28th day of life ( $P=0.22$; TABLE 1$)$. The systolic blood pressure, diastolic blood pressure, and mean blood pressure did not differ between preterm and term neonates $(P>0.01)$ (tABlE 1).

Echocardiography Compared with term neonates on the 28th day of life, preterm neonates had lower IVSd $(P=0.02)$, LVPWd $(P<0.001)$, and LVIDd ( $P=0.04$; TABLE 2). Preterm neonates had lower FS\% than term neonates after expected PDA closure $(P=0.01)$ and on the 28th day of life ( $P=0.002$; TABLE 2$)$. The LV cardiac output was higher in preterm than in term neonates after PDA closure $(P<0.001)$ and lower than in term neonates on the 28th day of life $(P=0.01$;

TABLE 1 Clinical characteristics of term and preterm neonates

\begin{tabular}{lll} 
Parameter & \multicolumn{1}{c}{$\begin{array}{c}\text { Preterm neonates } \\
(\mathrm{n}=89)\end{array}$} & $\begin{array}{c}\text { Term neonates } \\
(\mathrm{n}=29)\end{array}$ \\
\hline Gestational age at birth, wk, median (range) & $26(24-32)$ & $38(37-41)$ \\
\hline Male/female sex, $\mathrm{n}$ & $46 / 43$ & $18 / 11$ \\
\hline Mean birth weight, g, median (range) & $839(410-1920)$ & $3443(2700-4200)$ \\
\hline Cesarean delivery, $\mathrm{n}(\%)$ & $53(59)$ & $11(38)$ \\
\hline One-minute Apgar score, median (range) & $5(1-10)$ & $10(5-10)$ \\
\hline Five-minute Apgar score, median (range) & $7(2-10)$ & $10(7-10)$ \\
\hline PDA surgical ligation, $\mathrm{n}(\%)$ & $12(13.4)$ & 0 \\
\hline PDA pharmacological ligation only, $\mathrm{n}(\%)$ & $32(35.9)$ & 0 \\
\hline Prenatal or maternal steroids, $\mathrm{n}(\%)$ & $64(72)$ & 0 \\
\hline Surfactant therapy, $\mathrm{n}(\%)$ & $66(74)$ & 0 \\
\hline BPD (28th day of life), $\mathrm{n}(\%)$ & $50(56.2)$ & 0 \\
\hline BPD (36th week of PCA), $\mathrm{n}(\%)$ & $15(16.8)$ & 0 \\
\hline Heart rate, bpm, mean (SD) & $155.26(19.85)$ & $123.46(13.61)^{\mathrm{a}, \mathrm{c}}$ \\
\hline Systolic blood pressure, mm Hg, mean (SD) & & $149.14(15.60)^{\mathrm{b}}$ \\
\hline Diastolic blood pressure, mm Hg, mean (SD) & $81.78(12.51)$ & $77.70(10.39)^{\mathrm{a}}$ \\
\hline Mean blood pressure, mm Hg, mean (SD) & $45.83(10.62)$ & $46.25(15.34)^{\mathrm{b}}$ \\
\hline
\end{tabular}

a After PDA closure; $\quad$ b On the 28th day of life; $\quad$ c Significant difference with preterm neonates $(P<0.001)$

Abbreviations: BPD, bronchopulmonary dysplasia; PCA, postconceptional age; PDA, patent ductus arteriosus 
TABLE 2 Left ventricular function assessed by pulsed-wave Doppler echocardiography in preterm neonates at week 40 of postmenstrual age and term neonates after patent ductus arteriosus closure and on the 28 th day of life

\begin{tabular}{|c|c|c|c|c|c|}
\hline Parameter & $\begin{array}{l}\text { Term after PDA } \\
\text { closure }\end{array}$ & $P$ value ${ }^{a}$ & $\begin{array}{l}\text { Preterm } \\
\text { neonates }\end{array}$ & $P$ value ${ }^{a}$ & $\begin{array}{l}\text { Term on the 28th } \\
\text { day of life }\end{array}$ \\
\hline IVSd, cm & $0.52(0.34)$ & 0.15 & $0.39(0.08)$ & 0.02 & $0.47(0.18)$ \\
\hline LVPWd, cm & $0.38(0.23)$ & 0.7 & $0.33(0.09)$ & $<0.001$ & $0.46(0.12)$ \\
\hline LVIDd, cm & $1.48(0.39)$ & 0.03 & $1.67(0.38)$ & 0.04 & $1.86(0.26)$ \\
\hline $\mathrm{FS}, \%$ & $33.68(7.75)$ & 0.01 & $28.73(9.86)$ & 0.002 & $36.49(12.09)$ \\
\hline $\begin{array}{l}\text { LV cardiac output, } \\
\text { I/min } / \mathrm{kg}\end{array}$ & $0.36(0.11)$ & $<0.001$ & $0.56(0.19)$ & 0.01 & $0.76(0.28)$ \\
\hline MPI & $0.39(0.07)$ & $<0.001$ & $0.31(0.09)$ & 0.02 & $0.37(0.11)$ \\
\hline E wave, $\mathrm{cm} / \mathrm{s}$ & $50.10(9.57)$ & $<0.001$ & $78.20(13.51)$ & 0.02 & $69.20(14.17)$ \\
\hline A wave, $\mathrm{cm} / \mathrm{s}$ & $51.76(9.64)$ & $<0.001$ & $83.91(23.55)$ & 0.34 & 77.95 (18.94) \\
\hline E/A ratio & $0.99(0.20)$ & 0.94 & $0.98(0.22)$ & 0.3 & $0.92(0.20)$ \\
\hline
\end{tabular}

Data are presented as mean (SD). A P value of less than 0.05 was considered significant

a Pairwise comparisons with preterm neonates

Abbreviations: FS, fractional shortening; IVSd, interventricular septum thickness; LV, left ventricular; LVIDd, left ventricular internal diameter at end diastole; LVPWd, left ventricular posterior wall thickness; MPI, myocardial performance index; PDA, patent ductus arteriosus

TABLE 3 Tissue Doppler imaging of left ventricular function in preterm neonates at week $\mathbf{4 0}$ of postmenstrual age and term neonates after patent ductus arteriosus closure and on the 28th day of life

\begin{tabular}{llllll} 
Parameter & $\begin{array}{c}\text { Term after PDA } \\
\text { closure }\end{array}$ & Pvalue $^{\text {a }}$ & $\begin{array}{c}\text { Preterm } \\
\text { neonates }\end{array}$ & Pvalue $^{\text {a }}$ & $\begin{array}{c}\text { Term on the 28th } \\
\text { day of life }\end{array}$ \\
\hline$E^{\prime}$ wave & $7.13(1.60)$ & 0.02 & $8.37(2.14)$ & 0.99 & $8.36(2.28)$ \\
\hline$A^{\prime}$ wave & $6.46(1.47)$ & 0.01 & $8.11(2.51)$ & 0.85 & $8.36(2.68)$ \\
\hline$S^{\prime}$ wave & $5.33(1.17)$ & 0.83 & $5.45(1.18)$ & 0.18 & $5.86(1.04)$ \\
\hline E'/A' ratio & $1.14(0.31)$ & 0.98 & $1.15(0.48)$ & 0.59 & $1.08(0.40)$ \\
\hline
\end{tabular}

Data are presented as mean (SD). A $P$ value of less than 0.05 was considered significant.

a Pairwise comparisons with preterm neonates

Abbreviations: see TABLE 2

TABLE 2). The MPI was lower in preterm than in term neonates after PDA closure $(P<0.001)$ and on the 28th day of life ( $P=0.21$; tABLE 2$)$.

The indices of LV diastolic function, namely, the $\mathrm{E}$ and $\mathrm{A}$ waves, were higher in preterm than in term neonates after PDA closure and on the 28th day of life (E wave, $P<0.001$ to $P=0.02$; A wave, $P=0.34)$, but the $\mathrm{E} / \mathrm{A}$ ratio was similar in preterm and term neonates $(P>0.29$; TABLE2). Similarly, on TDI, the E' and A' waves were higher in preterm than in term neonates after PDA closure $(P=0.017$ and $P=0.01$, respectively), but the E'/A' ratios were similar in preterm and term neonates $(P>0.58$; TABLE 3$)$. The $S^{\prime}$ wave did not differ between preterm and term neonates after PDA closure and on the 28th day of life ( $P=0.83$ and $P=0.18$, respectively).
DISCUSSION In this study, we used several echocardiographic techniques to compare systolic and diastolic function of the LV between preterm and term neonates in the first month of life. We found that both systolic and diastolic function appeared normal in preterm neonates.

We measured MPI, which reflects both systolic and diastolic myocardial function. Previous studies reported that MPI decreases in the first weeks after birth, which corresponds to improved systolic and diastolic myocardial function. ${ }^{10,12,13}$ In our study, the MPI in preterm neonates at week 40 of PCA was significantly lower than in term neonates after PDA closure and on the 28th day of life. These findings suggest that the global function of the LV in preterm 
neonates was better than in term neonates in the first month of life. We suppose that preterm neonates in our study had a significantly better MPI than term neonates due to longer ex utero life, which allowed hemodynamic adaptations. However, in a previous study, in contrast to our current findings, we found similar MPI values in preterm neonates at week 40 of PCA (0.37) and term neonates after PDA closure (0.39) and on the 28th day of life (0.37). Moreover, MPI did not improve significantly in preterm neonates 4 weeks after birth (0.36). ${ }^{14}$ Similarly, in a study by Hirose et al, ${ }^{11} \mathrm{MPI}$ was similar in preterm neonates at week 40 of PCA and term neonates measured on the 28th day of life ( 0.44 vs 0.43 , respectively). Although we included neonates with and without bronchopulmonary dysplasia in our current study, we did not expect the dysplasia to affect MPI values in preterm neonates because LV MPI did not differ between preterm neonates with or without bronchopulmonary dysplasia in our previous study. ${ }^{11}$

In this study, FS\%, an indicator of systolic function, was lower in preterm neonates at week 40 of PCA than in term neonates after PDA closure and on the 28 th day of life. However, we suspect that the decreased FS\% in preterm neonates did not indicate impaired systolic function because other indicators of systolic function in preterm neonates ( $\mathrm{S}^{\prime}$ wave, $\mathrm{MPI}$ ) were not impaired in these children. We suspect that reduced FS\% in preterm neonates in our study was likely due to a significantly smaller myocardial size (IVSd, LVPWd) in preterm neonates. The M-mode imaging reflects the motion of only the anterior and posterior LV walls, and, in preterm neonates, the systolic motion of the anterior LV wall is less pronounced than that of the posterior and lateral LV walls; thus, FS\% may underestimate LV systolic function in these children. Similarly, in a study by Kozak-Barany et al, ${ }^{15}$ preterm neonates at 1 month had slightly reduced FS\%, but other parameters of systolic function were normal. Decreased FS\% of the heart muscle in preterm neonates could be associated with a reduced expression of contractile proteins in heart sarcomeres in preterm neonates, ${ }^{16}$ but this impairment seems to be compensated for, so that systolic function appears normal in preterm neonates. Moreover, in our study, although preterm neonates at week 40 of PCA had reduced FS\%, they had significantly higher cardiac output than term neonates after expected PDA closure, which could be explained by a substantially higher heart rate in the former (155 bpm vs $123 \mathrm{bpm}$, respectively). Indeed, when heart rate in term neonates increased on the 28th day of life, together with heart size, their cardiac output became significantly higher than that in preterm neonates.

In our study, preterm neonates had significantly higher values of E and E' waves than the other groups, but the E/A and E'/A' ratios were normal (close to 1 ) in both groups. Thus, the early diastolic function in preterm neonates at week 40 of PCA appeared normal. In contrast, Hirose et $\mathrm{al}^{17}$ found decreased diastolic function (E/A ratio) in preterm neonates; however, these investigators studied preterm neonates 4 weeks after birth, whereas we evaluated preterm neonates after, on average, 14 to 18 weeks of postnatal life (ie, after sufficient time for postnatal adaptation of diastolic heart function). Indeed, a previous study showed that LV diastolic function in preterm neonates improved to normal values in the first month of life..$^{15}$ In fetuses and preterm neonates, ventricular filling occurs mostly during atrial contraction because the stiff walls of the LV impair early diastolic ventricular filling through the mitral valve, which is reflected by pronounced A waves. Consequently, the $\mathrm{E} / \mathrm{A}$ ratio is lower in preterm neonates and fetuses (about 1.0:1) than in term neonates (about 1.1:1). ${ }^{18}$ Thus, in preterm neonates, diastolic dysfunction should be diagnosed only when the E/A ratio is lower than $0.6: 1$. In contrast, in adults, diastolic dysfunction is diagnosed already when the $\mathrm{E} / \mathrm{A}$ ratio is lower than $1 .{ }^{18}$

In our study, echocardiography was the only technique to assess myocardial function because other methods, particularly invasive measurements, cannot be used in neonates due to high risk. However, the strength of our study is a large group of preterm neonates $(\mathrm{n}=89)$ compared with previous studies involving much smaller groups ( $\mathrm{n}=16-29),{ }^{14,17}$ which compensates for the lack of invasive measurements.

In conclusion, systolic and diastolic function in preterm neonates at week 40 of PCA appears similar to that of term neonates after completing the neonatal period of life. Changes in the myocardium in preterm neonates depend on many factors and occur at different times than in term neonates; therefore, the optimal time points for comparisons of myocardial function between preterm and term neonates should be selected based on both PCA and chronological age, respectively.

\section{ARTICLE INFORMATION}

ACKNOWLEDGMENTS Editing support and language assistance were provided by Proper Medical Writing Sp. $z$ 0.0.

CONFLICT OF INTEREST None declared.

HOW TO CITE Bokiniec R, Własienko P, Szymkiewicz-Dangel J, et al. Echocardiographic analysis of left ventricular function in term and preterm neonates at week 40 of postconceptional life. Kardiol Pol. 2019; 77: 445-450. doi:10.5603/KP.a2019.0040

\section{REFERENCES}

1 Morton SU, Brodsky D. Fetal physiology and the transition to extrauterine life. Clin Perinatol. 2016; 43: 395-407.

2 Friedman AH, Fahey JT. The transition from fetal to neonatal circulation: normal responses and implications for infants with heart disease. Semin Perinatol. 1993; 17: 106-121.

3 Hooper SB, Te Pas AB, Lang J, et al. Cardiovascular transition at birth: a physiological sequence. Pediatr Res. 2015; 77: 608-614. 
4 Singh Y, Tissot C. Echocardiographic evaluation of transitional circulation for the neonatologists. Front Pediatr. 2018; 6: 140.

5 Wu TW, Azhibekov T, Seri I. Transitional hemodynamics in preterm neonates: clinical relevance. Pediatr Neonatol. 2016; 57: 7-18.

6 Hillman NH, Kallapur SG, Jobe AH. Physiology of transition from intrauterine to extrauterine life. Clin Perinatol. 2012; 39: 769-783.

7 Bensley JG, Moore L, De Matteo R, et al. Impact of preterm birth on the developing myocardium of the neonate. Pediatr Res. 2018; 83: 880-888.

8 Dice JE, Bhatia J. Patent ductus arteriosus: an overview. J Pediatr Pharmacol Ther. 2007; 12: 138-146.

9 Mathew B, Lakshminrusimha S. Persistent pulmonary hypertension in the newborn. Children (Basel). 2017; 4: 63.

10 Noori S. Pros and cons of patent ductus arteriosus ligation: hemodynamic changes and other morbidities after patent ductus arteriosus ligation. Semin Perinatol. 2012; 36: 139-145.

11 Bokiniec R, Własienko P, Borszewska-Kornacka M, Szymkiewicz-Dangel J. Evaluation of left ventricular function in preterm infants with bronchopulmonary dysplasia using various echocardiographic techniques. Echocardiography. 2017; 34: 567-576.

12 Noori S, Friedlich P, Seri I, Wong P. Changes in myocardial function and hemodynamics after ligation of the ductus arteriosus in preterm infants. J Pediatr. 2007; 150: 597-602.

13 Murase M, Morisawa T, Ishida A. Serial assessment of left-ventricular function using tissue doppler imaging in premature infants within 7 days of life. Pediatr Cardiol. 2013; 34: 1491-1498.

14 Bokiniec R, Własienko P, Borszewska-Kornacka MK, et al. Myocardial performance index (Tei index) in term and preterm neonates during the neonatal period. Kardiol Pol. 2016; 74: 1002-1009.

15 Kozák-Bárány A, Jokinen E, Saraste M, et al. Development of left ventricular systolic and diastolic function in preterm infants during the first month of life: a prospective follow-up study. J Pediatr. 2001; 139: 539-545.

16 Price $F$. Unique aspects of heart failure in the neonate. In: Shaddy $E$, ed. Heart failure in congenital heart disease: from fetus to adult. London: Springer; 2011: 21-42.

17 Hirose A, Khoo NS, Aziz K, et al. Evolution of left ventricular function in the preterm infant. J Am Soc Echocardiogr. 2015; 28: 302-308.

18 Riggs TW, Rodriguez R, Snider AR, Batton D. Doppler echocardiographic evaluation of right and left ventricular diastolic function in normal neonates. J Am Coll Cardiol. 1989; 13: 700-705. 